$\xi=-1$

\title{
Development of cognitive vehicle assistance and driver safety control management
}

\author{
B.Ankamma rao ${ }^{1 *}$, M.Harika Devi ${ }^{2}$, Krishna Veni Kommuri $^{3}$ \\ ${ }^{1}$ U.G Students, Dept. of E.C.S.E Koneru Lakshmaiah Education Foundation, Vaddeswaram, Guntur, Andhra Pradesh, India, 522502. \\ ${ }^{2}$ U.G Students, Dept. of E.C.S.E Koneru Lakshmaiah Education Foundation, Vaddeswaram, Guntur, Andhra Pradesh, India, 522502. \\ ${ }^{3}$ Dept. of E.C.M Koneru Lakshmaiah Education Foundation, Vaddeswaram, Guntur, Andhra Pradesh, India, 522502. \\ *Email: bodduluriankammarao5@gmail.com
}

\begin{abstract}
In this paper we present new approach for automobiles safety driving system involving the multiple sensor monitoring an area around vehicle and conveying alert the driver. There is some distance between two vehicles if apply break we can lose the control our vehicle. The proposed system works like that it has the four Ultrasonic sensors are fixed four sides of car they will detect the cars and obstacles within in the monitored area based on how much distance they are near to car and alert the driver via leds.an alcohol sensor fixed in the steering if he driver drink alcohol it will detect and switch off the vehicle ignition. The IR sensor monitors the eye blinks the driver. If the driver sleeps it makes ring the buzzer.
\end{abstract}

Keywords: Advance RISIC Machine; Alcohol sensors; IR sensor; Ultrasonic sensors,

\section{Introduction}

Embedded structures are planned to some specific errand, instead of be an all around utilize pc for various assignments. It persistent execution objectives that must should be met, for reason, for instance, prosperity and usability; others it's low or no execution requirements, empower the structure gear are enhanced to decrease costs.

Remote correspondence has transformed into a basic component for business things and a notable research subject inside the latest ten years. There are at present more PDA participations than wired-line enrollments. Generally, one area of business interest has been insignificant exertion, low-power, and short-isolate remote correspondence used for individual remote frameworks. "Innovation types of progress are giving tinier and all the more monetarily keen contraptions for organizing computational taking care of, remote correspondence, and a substantial gathering of various functionalities. The point when the vehicle is stolen, proprietor will give a call to the pack set in vehicle motor consequently stop which is demonstrated by the DC engine thusly sends the SMS to the approved individual demonstrating the correct area utilizing GPS. The vast majority of the controlling frameworks, today are inserted frameworks. The unpredictability of the frameworks may vary from to the next.In this venture another system in car innovation about how to keep 10 meter remove between one vehicle and another vehicle, so the vehicle don't crash or cause any activity issue. The point of the framework is to counteract mishaps predominantly because of not knowing the accompanying separation might be 10 meters between one vehicle and another vehicle.

This framework we have utilized ultrasonic sensors to distinguish any vehicle on both front and posterior of our vehicle. This framework is additionally utilized as a part of huge crane which is for the most part worked in harbor territory. In the event that the auto achieves 10-meter, green shading light will shine. At 8 meters remove yellow shading light will shine. When it achieves 5-meter separate red shading light will gleam. The separation is likewise shown to the vehicle driver.

From ultrasonic sensors are used for detecting the any vehicle is in danger distance to our vehicle it will alert by blinking of led lights. The alcohol sensors are fixed in steering before starting engine it asks the driver it blow the air into the sensor unit and its checks the alcohol content is there or not.

\section{System Architecture}

The system architecture are three features of driver vigilance. It each block in the diagram accounts for various purposes of the drivers. The main component is MSP430FR5969 is TEXAS Instrument Microcontroller featuring it is 16-bit RISIC architecture and ultralow power FRAM plate form. Up to $64 \mathrm{~kb}$ of Nonvolatile Memory. Wide supply voltage rang from $(1.8 \mathrm{~V}$ to $3.6 \mathrm{~V})$ It operate the CPU frequency range is up to $16 \mathrm{MHz}$. There are used three sensors purposes.

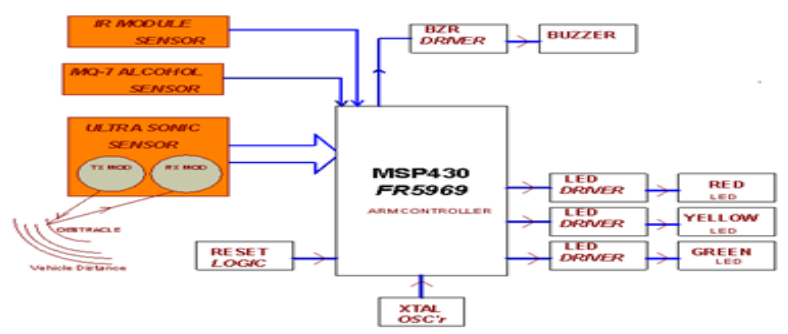

Fig:1 Block diagram 


\section{Hardware components}

\subsection{Ultrasonic sensor:}

To find the total of round-trip distance of sound wave. Round-trip means that the sound wave traveled 2 times the distance to the object before it can be detected by the sensor; it includes the 'trip' from the sonar sensor of object and the 'trip' from the object to the Ultrasonic sensor (after the sound wave bounced off the object). To find the distance to the object, simply divide the round-trip distance in half. So, it can be used to use to find the distance between the two cars and we can find the distance if are in so near to any car or obstacle that means danger distance or not. If we are danger distance it can intimate us. Ultrasonic sensors sends ultrasonic sound signals and receives those signals and calculate distance. If anyone sensor distance is less than 10meter distance automatically the LED' $s$ are glows for indicating the Alert to the driver based on distance, we use three types of led' s distance is equal to 10 meters a GREEN LED is turns ON state. If the distance is less than 8 centimeters automatically the Yellow LED turns ON state. If the distance is equal to 5 meters the RED LED is glows $\mathrm{ON}$ which means it is in dangerous position immediately alert the signals respectively. Driver under stands that his vehicle is in danger distance mode he can reduce the speed of the vehicle.

\subsection{IR sensor:}

An infrared sensor is an electronic instrument which is used to sense certain characteristics of its surrounding by either emitting and detecting infrared radiation. Infrared sensor is also capable to measure the heat being emitted by object and detecting motion.

This Eye Blink sensor is IR based. The Variation Across the eye will vary as per eye blink. If the eye is closed means the output is high otherwise output is low. This to know the eye is closing or opening position.

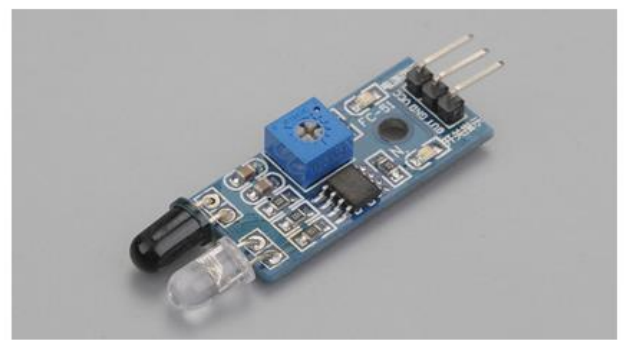

Fig: 2 IR sensor

\subsection{Alcohol sensor:}

An Alcohol sensor was used MQ-7. The MQ-7 is a Carbon Monoxide (CO) sensor. The semiconductor has gas sensing was $\mathrm{CO}$ concentrations in the air. It makes the detection of method cycle is high and low temperature and detect $\mathrm{CO}$ at low

Temperature. Alcohol sensor works the driver across the driving cases where the driver was drunk the car will be crash under the influence of alcohol causing damage and life. So, the proposed system is innovative to eliminate the such cases they would be monitoring the driver breath by placing it on steering part and the gas sensor will be detect and its lock the car engine. The gas sensor was detecting the LED will be blow. In this system we use a microcontroller interface with a gas sensor MQ-7.

\section{Flow Chart}

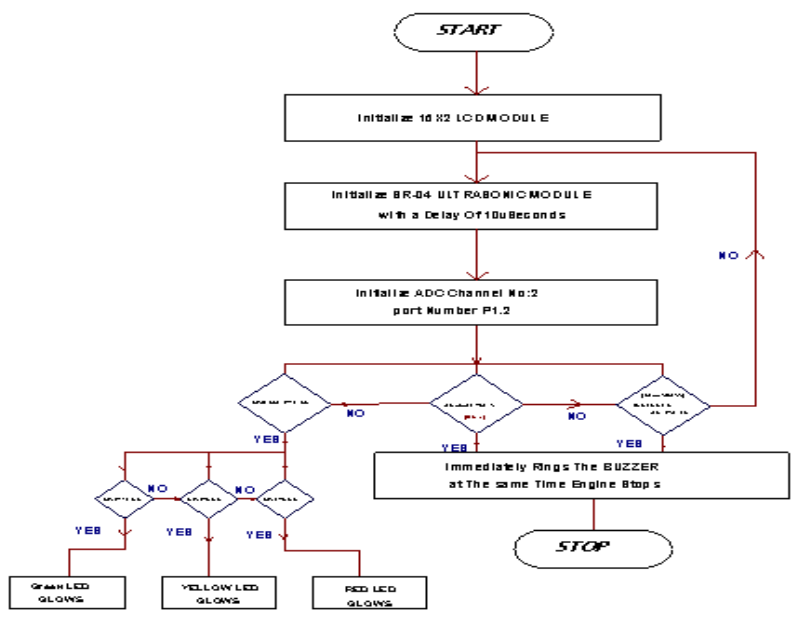

4.1 Theory of Flow Chart:

The whole process of the project is described in the flow chart. All the parts are connected to each other in serial order. The LCD (liquid crystal display) display is being initialized first. The next step is initializing HC SR-04 Ultrasonic Sensor with a delay of 10 Micro seconds. The next initialize 10-bit ADC channel number A5 with a port number is P1.5. After that in ultrasonic distance measurement there is three cases are derived.

CASE1: if ultrasonic senor distance is equal to 10 centimeters the GREEN LED is glows. Otherwise it goes to next case respectively.

CASE2: if ultrasonic senor distance is equal to 8 centimeters the YELLOW LED is glows. Otherwise it goes to next case respectively.

CASE3: if ultrasonic senor distance is equal to 10centi meters the RED LED is glows which means vehicle in danger condition, so the RED COLOR is indicating for danger situations.

The next parameter is alcohol if the driver is drink and drives automatically the MQ-7 Sensor detects and intimates the Alert BZR tones. If the alcohol level is exceeded greater than 50\% automatically the vehicle is stopped respectively.

The next parameter is IR MODULE especially it is used for driver in Drowsiness condition. Driver eye is closed in a very few seconds immediately the IR sensor detects and intimates the Alert tones to the driver for safety intimation. If more than three seconds eye is in closed position automatically the vehicle in stop condition.

If all sensor are working fine the loop will tracing each and every condition in closed loop program. If any one of these conditions are break immediately to alert the driver by glowing LED' $s$ and as well as BUZZER Sounds Respectively. Here in our project only demonstration purpose I use only on ultrasonic sensor but in real time approach 4 ultrasonic sensors are used for four sides of the vehicle if any sensor detects automatically it intimates information to the driver with the help of LED Glowing indications respectively. 


\section{Proposed System}

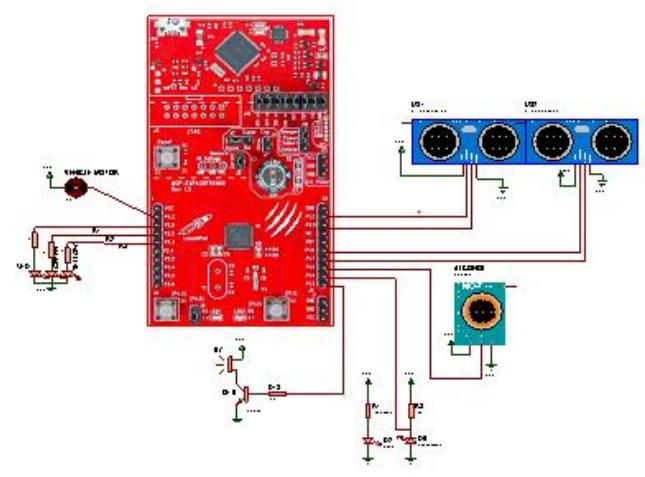

Fig:3 Circuit diagram

\subsection{Theory of circuit diagram:}

The Circuit diagram shows the whole process involved in the project. The entire circuit needs a power supply is $+5 \mathrm{~V}$ why because it is very low power consumption board. In this circuit I use two ultrasonic sensor modules. (HC-SR04) these are connected first ultrasonic TRIGGER pin is P1_2 and ECHO pin is P3_0 and the second ultrasonic sensor. If anyone sensor distance is less than 10-meter distance automatically the LED' $\mathrm{s}$ are glows for indicating the Alert to the driver based on distance, we use three types of led' s distance is equal to 10 meters a GREEN LED is turns ON state. If the distance is less than 8 centimetres automatically the Yellow LED turns ON state. If the distance is equal to 5 meters the RED LED is glows ON which means it is in dangerous position immediately alert the signals respectively.

In this project for alcohol detection I use MQ-7 Module for detection of alcohol sensor and it is connected to P1_5. This pin acts as a one of the ADC Channel A5. Why it is especially connected to ADC channel the alcohol sensor gives the analog data so hats why I use ADC for converting analog data into digital approach for interactive detection the driver how much percentage of deinked it is detected in percentage format respectively.

Next in this project I use IR MODULE for detection of drowsiness purpose the sensor gives the data output is digital when the driver is in drowsiness automatically his eyes are closed at that time the IR sensor is gives the logic 1 to the controller which means the driver in unconscious state at that time immediately the BUZZER is activated the buzzer is connected to pin number P1_3 Respectively.

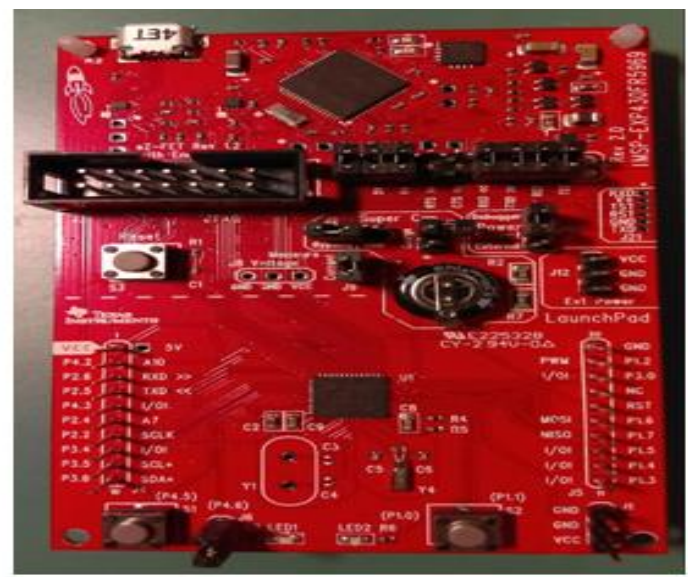

Fig:4: MSP430FR5969 TEXAS Instrument Microcontroller

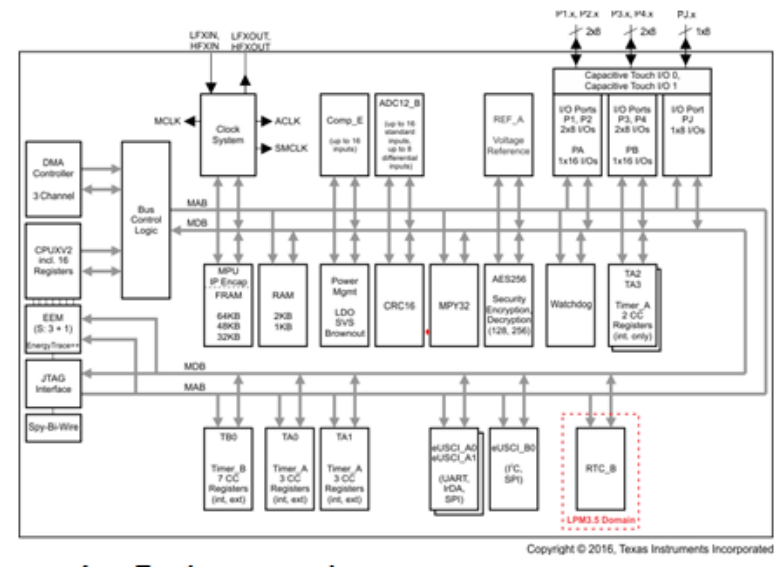

Fig:5 Functional Block Diagram

\subsection{Other Power Supply Pins 5V and 3.3V pins}

These pins can be used to provide power to sensors andmodules5V and $3.3 \mathrm{~V}$ pins. These pins can be utilized to give energy to sensors and modules while associating it to. MSP430FR5969 Controller IOREF This stick gives voltage reference which the microcontroller works.

\section{Power Configuration Layout}

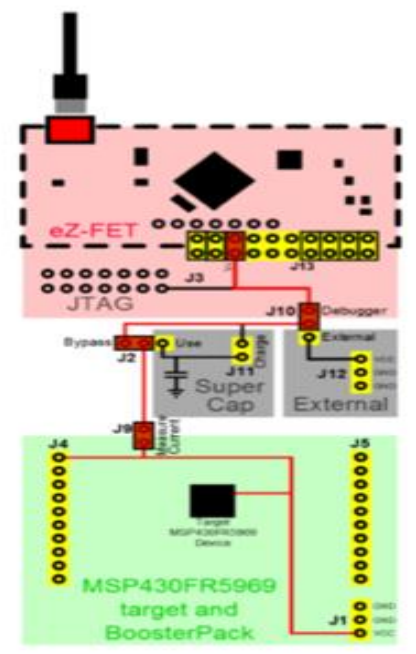

6.1 Ultrasonic sensor monitoring unit:

In this system we use four ultrasonic sensor modules. These are connected first ultrasonic sensors TRIGGER pin and ECHO pin and these pins are connected to MAP board. Ultrasonic sensors are send ultrasonic sound signals and receives those signals and calculate distance. If anyone sensor distance is less than 10meter distance automatically the LED' $s$ are glows for indicating the Alert to the driver based on distance, we use three types of led' $s$ distance is equal to 10 meters a GREEN LED is turns ON state. If the distance is less than 8 centimetres automatically the Yellow LED turns ON state. If the distance is equal to 5 meters the RED LED is glows ON which means it is in dangerous position immediately alert the signals respectively. Driver under stands that his vehicle is in danger distance mode he can reduce the speed of the vehicle.

\subsection{Alcohol detecting unit:}

Alcohol sensor works the driver across the driving cases where the driver was drunk the car will be crash under the influence of alcohol causing damage and life. So the proposed system are 
innovative to eliminate the such cases they would be monitoring the driver breath by placing it on steering part and the gas sensor will be detect and its lock the car engine. The gas sensor was detecting the LED will be blow. In this system we use an microcontroller interface with an gas sensor MQ-7. The system was push button to start the engine. If alcohol is detected at the time of starting the engine the engine des not start at all. If the gas sensor is detected after the car engine starting, the propose system locks the car engine at that time only. The driver was safe, and the alcohol content was set certain range only, the range was crossed the car engine was automatically locked.

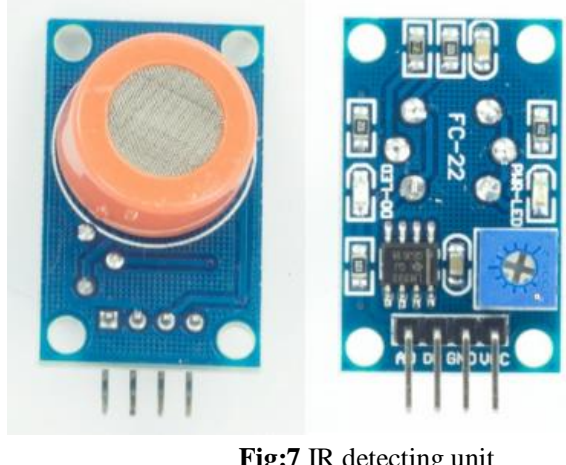

Fig:7 IR detecting unit

IR senor works like When Infrared Transmitter light emitting diode. If they are called IR LED. The IR LED are looks likes normal LED, radiation emitted by the invisible to the human eye. Infrared receivers are also called as infrared sensors are detected by radiation from an IR transmitter. When infrared Photodiodes are differing from normal photo diodes are detect only infrared radiation.

It will have fixed in car particular angle which monitor the eye blinks. When the eye blinks are less, or no eye blinks the infrared radiation is detected by the by the receiver it will signal to microcontroller that will give the signal to buzzer it will ring due to that sound the driver will weak up.

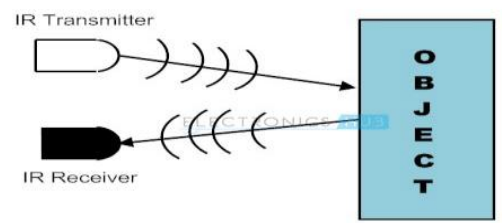

Fig :8 IR principle of operation

\subsection{The development software module:}

The MSP430FR5969 Integrated Development Environment - or Software consists of a word processor to compose code here we are using the software fir $3^{\text {rd }}$ party user is Energia 1.6.10 Version" when compared to IAS, GCC compiler which is more advantage and flexible for writing $\mathrm{C}, \mathrm{C}++$ programs and one more important point there is no need to install software it is simply Plug and run the software and which supports all operating systems like LINUX, WINDOWS, MAC respectively.

\subsubsection{Software Requirements:}

Energia 1.6.10 Version for $3^{\text {rd }}$ party IDE it is flexible and no need to install it and one more advantage is which support all operating systems (OS) Respectively.

This section includes all specifications and features of all MSP debug probes. The debug probe hardware and different debug mode configuration and setting are descried.
As shown below Figure is an overview of the MSP ecosystem showing the relations between IDE and debug probe and the MSP 430 fr 5969

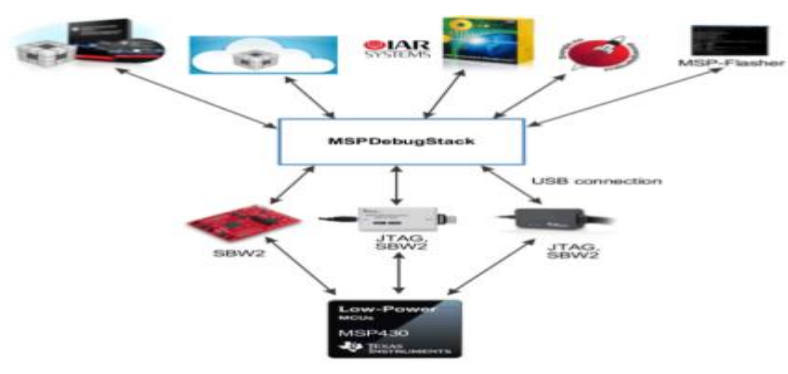

Fig9: MSP ECO SYSTEM

\subsection{AC Analog Input Module:}

Protection relays are designed based on the application type generator protection, transformer protection, motor protection, feeder protection, transmission and distribution protection etc. Relays that are modular are designed to have multiple functional modules. The AC analog input module performs the data acquisition function that consists of various sensors to measure voltage and current, signal conditioning circuitry, a data acquisition system, and a host interface. TI reference designs and ICs help:

\subsection{AC Drive Control Module:}

Integrated circuits and reference designs for AC drive control module systems, complete with schematics, test data and design files:

Power supply solutions for point of load with high efficiency, lowest output voltage ripple, tracking and monitoring, and small form factor.High performance analog solutions for low latency, high accuracy current, voltage and position sensing.Real-time embedded processing solutions with integrated motor control, specific low latency peripherals to reduce system cost and boost AC Drive performance.Broad variety of isolated and non-isolated industry standard interfaces with software programmable multiprotocol industrial Ethernet and field bus solutions.

\subsubsection{AC Drive Wired \& Wireless Communication:}

Integrated circuits and reference designs for AC drive wired and wireless systems, complete with schematics, test data and design files:Industry 4.0 solutions with a broad range of high-speed, low latency wired/wireless solutions.Broad variety of isolated/nonisolated robust industry standard interface solutions based on Ethernet, RS-485, CAN, LVDS, and more. Processors with software are programmable multiprotocol industrial Ethernet and field bus solutions. Analog input/output solutions that increase performance and reduce board space for industry standard analog I/O. Solutions for all major wireless standards including support for antenna layout and protocol stack, reducing time to market 
MSP430FR5969 HARDWARE LAYOUT:

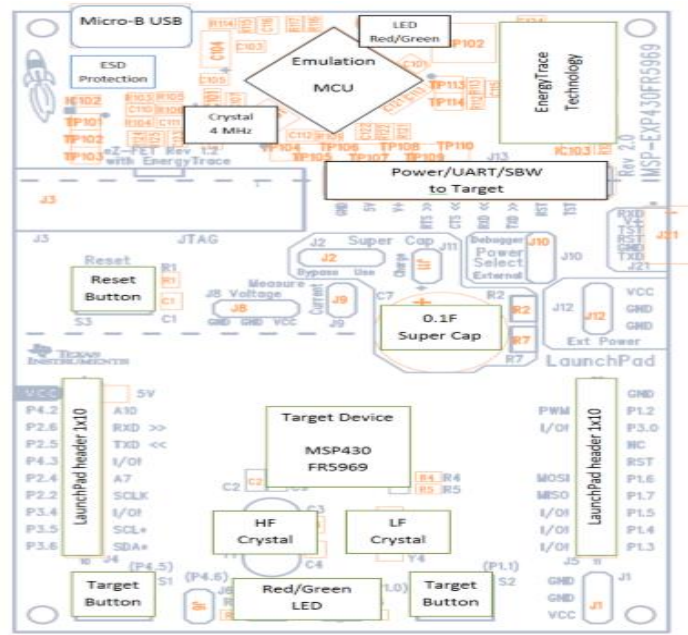

Fig 10 HARDWARE DIAGRAM OF MSP430FR5969 CONTROLLER

MSP430FR5969 BLOCK DIAGRAM:

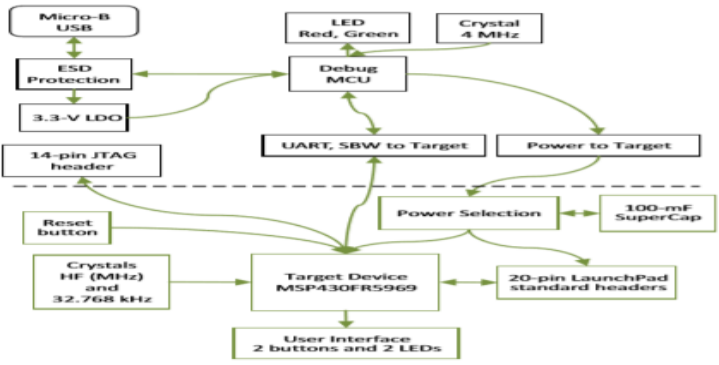

Fig11 BLOCK DIAGRAM OF MSP430FR5969 CONTROLLER

\section{Software Implementation}

\subsection{Energia 1.6.10 V IDE}

The MSP430FR5969 Integrated Development Environment or Software consists of a word processor to compose code here we are using the software fir $3^{\text {rd }}$ party user is Energia 1.6.10 Version when compared to IAS, GCC compiler which is more advantage and flexible for writing $\mathrm{C}, \mathrm{C}++$ programs and one more important point there is no need to install software it is simply Plug and run the software and which supports all operating systems like LINUX, WINDOWS, MAC respectively.

Projects that are executed using Energia software IDE as called draws. These representations are made the content manager and the spared are record augmentation. The edit manager has high light for glue and for supply content. The message region give input while sparing and trading and further more show mistakes. When toolbar enable to confirm and transferring the program.

\subsection{Software}

The required configuration is done in Software so we can dump program in Arduino

Open the Energia Software by double clicking the Energia icon (energia.exe)

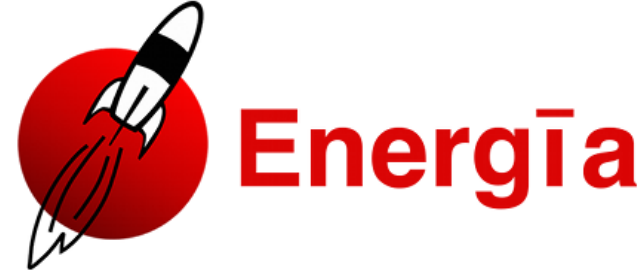

Fig12 Energia ICON

Connect the MSP430FR5960 board to the PC then a com will appear select the com in tools i.e. TOOLS>

$\mathrm{PORT}>\mathrm{COM}$ and click on it.

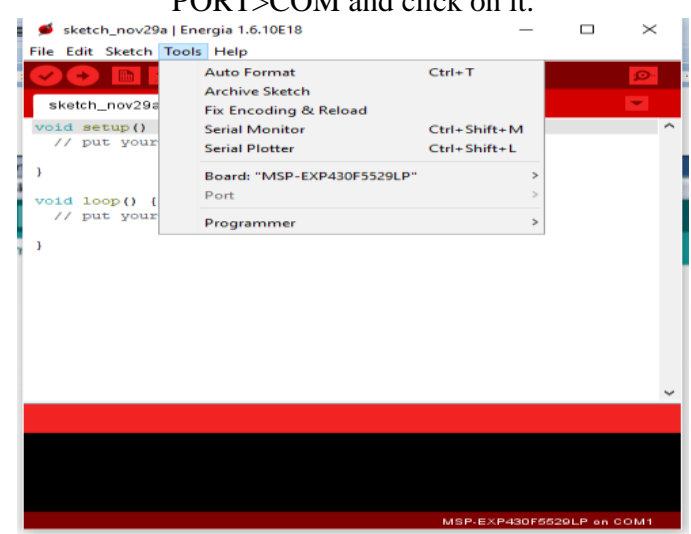

Fig13 STEP 1 IN COMPILING THE PROGRAM

Select the entry in the Tools greater than Board menu that corresponds to MSP430FR5969 Board. Our board is LANCHPAD so we have selected LANCHPAD here

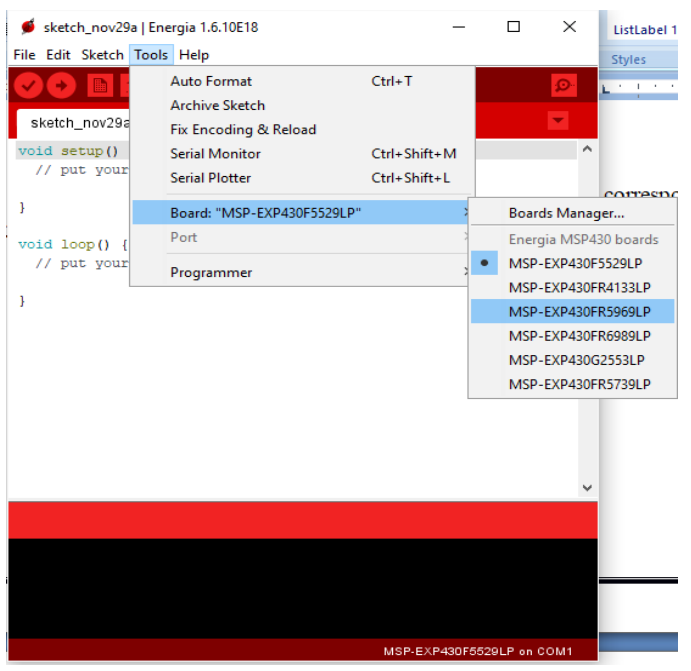

Fig STEP 2 In Compiling the Program

Select the programmer as rf 2500 as ISP 


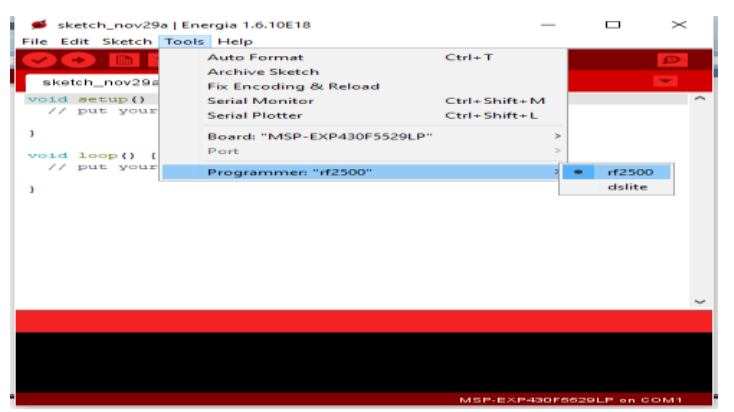

Fig 14 step 3 In compiling the program

\section{Software Outputs}

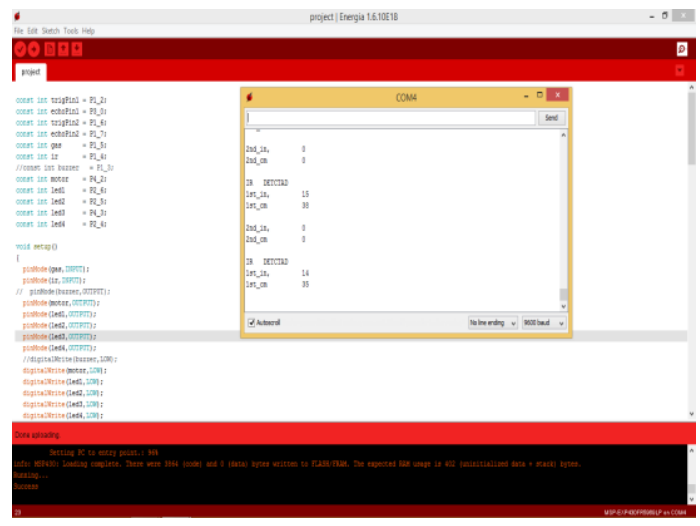

Fig 15 MSP430FR5969 BOARD INTERFACING SR-04

\section{ULTRASONIC SENSOR}

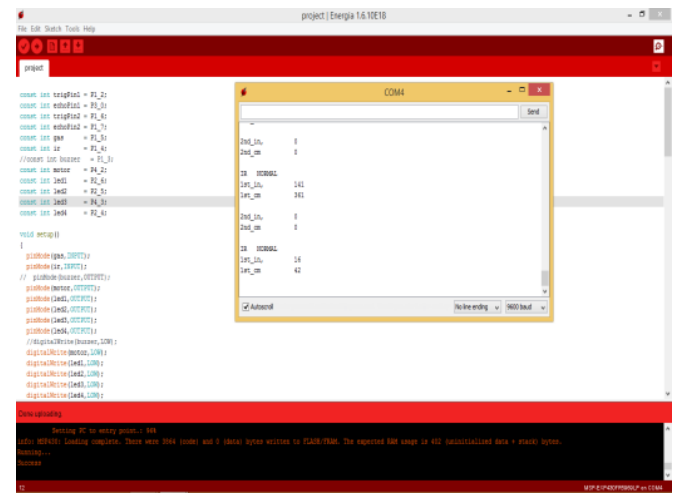

Fig16 MSP430FR5969 BOARD INTERFACING MQ-7 \& IR MODULE

\section{Hardware Results}

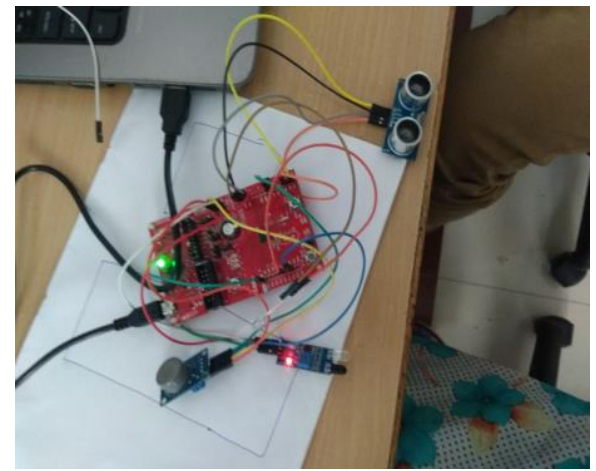

\section{ULTRASONIC SENSOR}

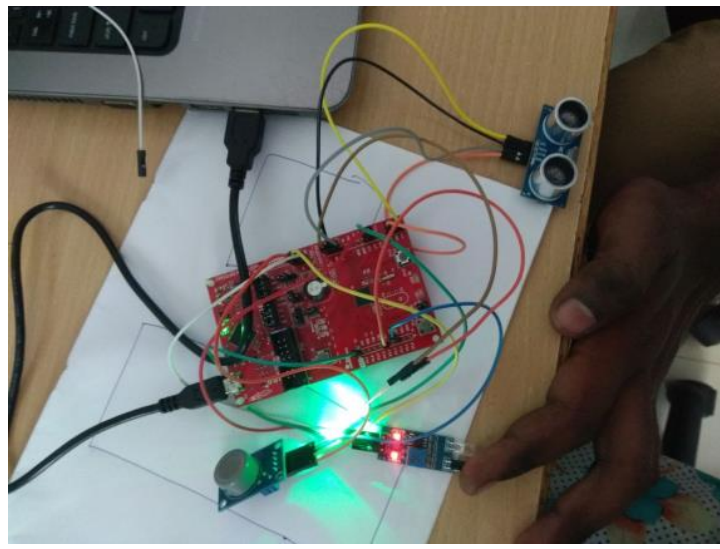

Fig 18 MSP430FR5969 BOARD INTERFACING MQ-7 \& IR MODULE.

\section{Conclusion}

The main advantage of the system is controlling the accidents due to the over speed of the vehicles. And alert if any one vehicle towards near to the vehicle simply indicates with the help of LED displays. If automatically detects drives takes alcohol in interactive procedure. The main advantage of this system is that the vehicle in safety and the driver even in drowsiness condition it is also detect alcohol levels. Finally we conclude that our vehicle is under conditionally safety and powerful sensing conditions respectively procedure.

\section{References}

[1] A wireless sensor based driving assistance for auto mobiles by Fred Yu, Bozena Kaminska, and Pawel Gburzynski School of Engineering Science, Simon Fraser University

[2] Driver safety awareness and assistance system for cognitive vehicle control by Janani.N, Saranya.n ISBN No. 978-1-4799-39145/14/\$31.00 @2014 IEEE

[3] A sensor network based intelligent control system for traffic distance by zhejun he, jiang zhang, peng xu, 978-0-7695-5079-4/13 \$26.00 @ 2013 IEEE DOI 10.1109/ISCID.2013.88

[4] Hang-Bang Kang, "Various Approaches for Driver and Driving Behavior Monitoring: A Review," Catholic Univ of Korea, ICCVW 14147905@20131EEE.

[5] Development of web-based collaboration framework for the simulation of embedded system by woong yang, soo-hong lee, young zhu jin, hyun tae hwang. Journal of computational design and engineering 3(2016)363-369

[6] Raj at Garg, Vikrant Gupta, ieeet Agrawal "A Drowsy Driver Detection and Security System" 9781-4244-3941-6/09/\$25.00 @2009 IEEE, Department of Electronics and Communication VITUniversity.

[7] N. Sueharu et al. A Study of the Driving Support System Based on Drivers Behavior Analysis, vol. 57, no. 12, 2003, pp. 102-107, Journal of the Society of Automotive Engineers of Japan,

[8] H. Jula, E. B. Kosmatopoulos, and P. A. Ioannou, "Collision Avoidance Analysis for Lane Changing and Merging, Vol. 49, No. 6, pp 2295-2308, Nov. 2000, IEEE Transactions on Vehicular Technology. 